\title{
Repeatability of different methods to collect in everyday clinics the sagittal profile of patients with adolescent idiopathic scoliosis Fabio Zaina ${ }^{1}$, Stefano Negrini ${ }^{* 1}$, Michele Romano ${ }^{1}$ and Angelo Aulisa ${ }^{2}$
}

\author{
Address: ${ }^{1}$ ISICO (Italian Scientific Spine Institute), Via Carlo Crivelli 20, 20122 Milan, Italy and ${ }^{2}$ Rome, Italy \\ Email: Stefano Negrini* - stefano.negrini@isico.it \\ * Corresponding author
}

from 4th International Conference on Conservative Management of Spinal Deformities

Boston, MA, USA. 13-16 May 2007

Published: 12 October 2007

Scoliosis 2007, 2(SuppI I):S44 doi:I0.II86/I748-7|6I-2-SI-S44

This abstract is available from: http://www.scoliosisjournal.com/content/2/SI/S44

(c) 2007 Zaina et al; licensee BioMed Central Ltd.

\section{Objective}

Assessing the repeatability of different methods to collect in everyday clinics the sagittal profile of patients with adolescent idiopathic scoliosis to identify the best one to be used.

\section{Study design}

We performed two studies. In the first one, in a medical setting, 61 patients were consecutively evaluated by two examiners (inter-observers), while one performed a second time the evaluation after 5 minutes (intra-observer); the evaluations included: sagittal and frontal distances from the plumbline of C7, sagittal distance of T12 and L3, D'Osualdo's Arcometer measurement. In the second study, in a physiotherapist setting, the sagittal distances (cervical, C7, thoracic, lumbar, sacral) have been evaluated consecutively using two different tools to identify verticality: a plumbline or a laser. Repeatability has been evaluated according to Bland and Altman, so to identify the limits of variation clinically significant.

\section{Results of the first study}

See Table 1.

\section{Results of the second study}

We verified that the usage of the laser in a physiotherapist setting reduced the errors due to positioning of the plumbline and movements of the hands during measurement of multiple data.

\section{Conclusion}

These results give the limits during measurements in a clinical setting of parameter that are routinely collected by some clinicians. $1 \mathrm{~cm}$. is the minimum change to be considered clinically significant between two examinations for C7, while this is 1.5 for T12 and L3, but only when measurements are taken always by the same treating physicians.

Table I: Results of the first study.

\begin{tabular}{|c|c|c|}
\hline Parameter & Intra-observer & Inter-observer \\
\hline Sagittal distance of C7 from the plumbline $(\mathrm{cm})$. & 0.9 & 1.7 \\
\hline Sagittal distance of DI 2 from the plumbline $(\mathrm{cm})$. & 1.3 & 1.9 \\
\hline Sagittal distance of L3 from the plumbline $(\mathrm{cm})$. & 1.2 & 2.2 \\
\hline D'Osualdo Arcometer (calculated $\left.{ }^{\circ} \mathrm{Cobb}\right)$ & 7 & 15 \\
\hline $\operatorname{ATR}\left({ }^{\circ}\right)$ & 1.4 & \\
\hline Height of the hump (mm.) & 2.1 & \\
\hline Frontal distance of $\mathrm{C} 7$ from the plumbline $(\mathrm{cm})$. & I.I & \\
\hline
\end{tabular}

